\title{
Myeloma-Related Disorders in Cats Commonly Present as Extramedullary Neoplasms in Contrast to Myeloma in Human Patients: 24 Cases with Clinical Follow-up
}

\author{
Paul J. Mellor, Sean Haugland, Sue Murphy, Ken C. Smith, Andrew Holloway, Joy Archer, \\ Roger M. Powell, Gerry A. Polton, Severine Tasker, David McCormick, Michelle E. Tempest, \\ Pauline E. McNeil, Timothy J. Scase, Chris D. Knott, Ugo Bonfanti, Elizabeth J. Villiers, \\ David J. Argyle, Michael E. Herrtage, and Michael J. Day
}

\begin{abstract}
Background: Myeloma-related disorders (MRD) are rare neoplasms of plasma cells. Published case reports describe a diversity of clinical presentations with confusing terminology and diagnostic criteria as a consequence of the assumption that MRD in cats are analogous to those in dogs or humans.

Objective: The aim of the study was to describe clinical, clinicopathologic and imaging findings, response to treatment, survival and possible associations with other diseases or vaccination in a large case series. A priori hypotheses were that cats with MRD commonly present with extramedullary involvement and uncommonly have radiographic bone lesions, in contrast to human patients.

Animals: Twenty-four cats with MRD confirmed by cytology or histopathology and immunohistochemistry.

Method: A multicenter retrospective study was performed.

Results: Two types of clinical presentation were observed. The first group $(\mathrm{n}=17)$ had neoplasia involving abdominal organs, bone marrow, or both. All developed systemic clinical signs and paraproteinemia. Five of 7 cats that received chemotherapy improved clinically or had decreased serum globulin concentration (median survival, 12.3 months; range, 8.5 22 months). The second group comprised 7 cats with skin masses, 2 of which were paraproteinemic and developed rapidly worsening systemic signs. In cats without systemic signs, excision of the skin masses appeared to be associated with prolonged survival (up to 2.4 years). Cats with MRD commonly presented with extramedullary involvement (67\%), versus humans with MRD $(5 \%)(P<.001)$, and uncommonly presented with radiographic bone lesions $(8 \%)$ versus humans with MRD $(80 \%)$ $(P<.001)$
\end{abstract}

Conclusions: Radiographic bone lesions are uncommon in cats with MRD and extramedullary presentation is common, relative to human myeloma.

Key words: Cat; Extramedullary plasmacytoma; Hyperglobulinemia; Monoclonal gammopathy; Multiple myeloma; Paraproteinemia; Plasma cell tumor.

$\mathbf{M}$ yeloma-related disorders (MRD) arise from the neoplastic transformation of plasma cells or immunoglobulin-secreting B lymphocyte precursors. The classification of MRD is outlined in Fig 1. MRD are rare in the cat, accounting for $0.003-0.1 \%$ of all malignancies reported in 2 large case series. ${ }^{1,2}$ By contrast, MRD account for $\sim 1 \%$ of all tumors in $\operatorname{dogs}^{3}$ and $1-2 \%$ of all neoplasia in humans. ${ }^{4}$

From the Department of Veterinary Medicine, University of Cambridge, Cambridge, UK (Mellor, Haugland, Holloway, Archer, Powell, Villiers, Herrtage); and the Animal Health Trust, Lanwades Park, Kentford, UK (Murphy, Smith, Scase); and Davies Veterinary Specialists, Hitchin, Hertfordshire, UK (Polton); and the School of Clinical Veterinary Science, University of Bristol, Langford, UK (Tasker, Day); and Runnymede Hill Veterinary Hospital, Tite Hill, Egham, Surrey, UK (McCormick); and the Cambridge University Hospitals NHS Foundation Trust, Addenbrooke's Hospital, Cambridge, UK (Tempest); and Finn Pathologists, Weybread, Diss, Norfolk, UK (McNeil, Knott); and Clinica Veterinaria Gran Sasso, 26-20131, Milano, Italy (Bonfanti); and the Royal (Dick) School of Veterinary Studies, University of Edinburgh, Midlothian, UK (Argyle).

Reprint requests: Paul Mellor, Department of Veterinary Medicine, University of Cambridge, Cambridge, UK; e-mail: p.j.mellor.02@cantab.net.

Submitted September 21, 2005; Revised April 3, 2006; Accepted May 28, 2006.

Copyright $(\odot) 2006$ by the American College of Veterinary Internal Medicine

0891-6640/06/2006-0014/\$3.00/0
Multiple myeloma (MM) is the archetypal MRD, and the diagnostic criteria in dogs and humans include the presence of neoplastic plasma cells in bone marrow accompanied by 1 or more of the following: (1) serum paraproteinemia, (2) immunoglobulin light chain (Bence-Jones) proteinuria, or (3) osteolytic bone lesions. ${ }^{5,6}$ Radiographically detectable bone lesions are observed in $80 \%$ of all human patients with an MRD. ${ }^{7}$

Extramedullary plasmacytoma (EMP) is defined as neoplastic plasma cell formation in soft tissue without primary evidence of bone marrow involvement and can be divided into cutaneous (CEMP) and noncutaneous (NCEMP) forms. ${ }^{8-10}$ Thirteen cats with EMP and clinical data have been reported in the veterinary literature, although a variety of descriptors were used including "plasma cell tumors" and "myeloma." Twelve cats had clinical features typically seen in MM. This finding differs from the situation observed in humans with MRD, where the majority of patients with CEMP and some patients with NCEMP have benign tumors. Overall, $<5 \%$ of humans affected by MRD have extramedullary involvement as a presenting manifestation. ${ }^{11,12}$

MRD is the most common cause of monoclonal paraproteinemia in cats accounting for 6 of $14(43 \%)$ reported cases. ${ }^{13}$ In the cat, the pathophysiology in MM and EMP is similar, typically resulting in the production of a serum paraprotein which may lead to hyperviscosity syndrome (HVS). ${ }^{14-17}$ Renal dysfunction may result from reduced perfusion, amyloidosis, increased filtration of 
1) Multiple myeloma (MM)

2) Cutaneous extramedullary plasmacytoma (CEMP)

3) Non-cutaneous extramedullary plasmacytoma (NCEMP)

4) Solitary plasmacytoma of bone (SPB)

5) IgM macroglobulinaemia (Waldenströms)

6) Immunoglobulin-secreting lymphoma

7) Immunoglobulin-secreting leukaemia

8) Plasma cell leukaemia

Fig 1. Classification of feline myeloma-related disorders.

immunoglobulin light chains, hypercalcemia, or tumor infiltration. ${ }^{8,13,18,19}$ Nonregenerative anemia is common. ${ }^{15,20}$

Little information regarding MRD in cats is available. Historically, the diagnostic criteria used in humans or dogs with MM have been applied to the cat, ${ }^{20-22}$ despite the fact that many of the case reports of affected cats describe features that differ from those in dogs or humans. In this study, the a priori hypotheses were that cats with MRD commonly present with extramedullary involvement and uncommonly have radiographic bone lesions, in contrast to the situation observed in human patients. The aims of the study included description of the clinical, clinicopathologic and imaging features, as well as treatment and outcome in a large series of cats with MRD confirmed by cytology, or by histopathology and immunohistochemistry. A secondary aim of the study was to examine for any association between MRD and other diseases by assessment of medical records and from owner follow-up.

\section{Materials and Methods}

Cats were identified by database search or by direct contact with veterinarians, at all major public and private UK referral centers and laboratories (see Acknowledgments section). A spectrum of disciplines (oncology, pathology, clinical pathology, internal medicine, ophthalmology, soft tissue surgery, orthopedics, dermatology, and neurology) was included in an attempt to avoid selection bias. Search terms were plasma cell neoplasia, myeloma, plasmacytoma, macroglobulinemia, hyperglobulinemia, gammopathy, and paraproteinemia. Cases were derived from the period 1990 to 2004. Permission for use of the clinical records, clinicopathologic data, radiographs, and cytologic and histopathologic material was obtained from referral and primary veterinarians and their clients. The inclusion criteria were the following:

(1) a report of cytologic or histopathologic confirmation or both, of a tumor with plasma cell or plasma cell-like morphology or, alternatively, lymphoid morphology as long as the tumor was accompanied by hyperglobulinemia $(>5.2 \mathrm{~g} / \mathrm{dL}$ ) attributable to a paraproteinemia (as evidenced by serum protein electrophoresis),

(2) confirmation of the reported morphologic diagnosis by review of the original cytologic slides, histopathologic sections or both. All histopathologic specimens were evaluated with anti-immunoglobulin antibody immunohistochemistry against $\alpha, \beta$, and $\mu$ heavy chain as well as $\kappa$ and $\lambda$ light chain isotypes. ${ }^{\mathrm{a}, \mathrm{b}}$ Specimens that demonstrated expression of both $\kappa$ and $\lambda$ light chain isotypes were diagnosed as reactive (ie, not neoplastic) and were excluded.
(4) complete follow-up information from either the clinical records or directly from the client.

Reports for 56 cats were obtained, of which 24 met the inclusion criteria. Original cytologic slides $(n=16$ positive specimens from $n$ $=13$ cats) were reviewed by a joint panel of cytopathologists (JA, EJV, RMP, PJM). Histopathologic samples $(\mathrm{n}=47$ positive specimens from $n=17$ cats) were reviewed separately by a joint panel of histopathologists (SH, KCS, TJS, PEM). Detailed cytologic, histopathologic, and immunohistochemical features will be reported elsewhere. Serum (SPE) and urine (UPE) protein electrophoresis was conducted in 19 and 16 cats, respectively. Original SPE and UPE traces were retrieved in 14 and 6 cases, respectively, and reviewed by a single clinical pathologist (JA). Serum paraprotein immunoglobulin type was assessed by agar gel immunodiffusion assay by a single pathologist (MJD). ${ }^{\text {a }}$ Thirteen cats were radiographed. All views were retrieved and reviewed by a single radiologist (AH). A complete past medical history was obtained for all 24 cats from the owner or the primary veterinarian(s) and referral veterinarian. Records were examined for evidence of (1) feline immunodeficiency virus (FIV), feline leukemia virus (FeLV), and feline infectious peritonitis virus (FIP) status; (2) presence of historic and concurrent diseases associated with chronic antigenic stimulation (eg, severe gingivitis, cholangiohepatitis); (3) vaccination history; and (4) nonplasma cell neoplasms.

\section{Statistical Analysis}

The differences in proportions of cases between cats and that reported in humans with regard to (1) extramedullary involvement and (2) radiographically detectable bone lesions at initial presentation were examined by means of 2 -sided $z$-tests. Differences in mean serum total globulin concentrations were compared by means of Student's paired $t$-test. Significance was set at $P<.05$

\section{Results}

\section{Signalment and Clinical Findings}

The median age was 12 years (range, 4-18 years), $84 \%$ were domestic short-haired cats, and there was an equal sex distribution ( $42 \%$ neutered females, $8 \%$ intact females, $50 \%$ neutered males). Presentation for vague clinical signs such as decreased appetite, weight loss, or lethargy was common (Table 1). A palpable abdominal mass (eg, hepatomegaly or splenomegaly) was common. A single cat with hepatomegaly also had oral mucosal plasma cell lesions (small nodular lesions at the fauces). Bleeding abnormalities (hyphema, melena, oral bleeding) were recorded in 4 other cats. No cat had a history of recurrent or atypical bacterial infections. Seven cats presented with cutaneous or subcutaneous masses, and 3 of them had multiple masses. Head and neck masses were identified in 5 cats. Other locations included the thorax and limbs. Pain was suspected in 1 cat only, localized to a soft tissue mass on a limb. Three apparently clinically normal cats were discovered to have hyperglobulinemia as incidental findings after routine blood tests (Table 1), although all 3 cats subsequently developed clinical signs.

\section{Clinicopathologic Data}

Initial investigations included both hematology and serum biochemistry in 22 of the 24 cats. Nine cats had 
Table 1. Historical and presenting clinical signs in 24 cats with myeloma-related disorders.

\begin{tabular}{lcc}
\hline & No. of Cases & Frequency (\%) \\
\hline Decreased appetite & 10 & $42 \%$ \\
Weight loss & 8 & $33 \%$ \\
Lethargy & 8 & $33 \%$ \\
Skin/subcutaneous masses & 7 & $29 \%$ \\
Palpable abdominal organ enlargement (usually hepatomegaly or splenomegaly) or a mass & 6 & $25 \%$ \\
Vomiting/diarrhea/dyschezia & 6 & $25 \%$ \\
Lameness/hindlimb weakness/ataxia & 3 & $17 \%$ \\
Polydipsia/polyuria & 3 & $13 \%$ \\
Central neurologic signs (eg, seizures, tremors) & 3 & $13 \%$ \\
Fundic changes (eg, hemorrhage, retinal vessel tortuosity, retinal detachment) & 3 & $13 \%$ \\
Bleeding abnormalities (eg, oral bleeding, melena, hyphema) & 3 & $13 \%$ \\
Incidental discovery of hyperglobulinemia on routine bloods & & $13 \%$ \\
\hline
\end{tabular}

non-regenerative anemia (NRA) at initial presentation and follow-up hematological examinations revealed that 11 cats developed NRA during the course of their disease (defined as a hematocrit $[\mathrm{HCT}]<0.25 \mathrm{~L} / \mathrm{L}$ recorded on 2 blood samples taken $>5$ days apart with no evidence of increasing HCT and polychromasia, no reticulocytosis after new methylene blue staining or both). Rare circulating plasma cells were noted in 1 cat that had marked $(>70 \%)$ bone marrow infiltration. Tests of hemostatic function were not conducted in the 4 cats with historical or clinical signs of bleeding. Of 7 cats with azotemia, 5 had renal insufficiency (defined as a serum creatinine concentration $>1.92 \mathrm{mg} / \mathrm{dL}[>$ $170 \mu \mathrm{mol} / \mathrm{L}]$ in combination with urine specific gravity $<1.035$ at any time point in the course of the disease, or recurrently high serum creatinine concentrations at $\geq 2$ time points with a history of polyuria and polydipsia). Increased liver enzyme activity (alanine aminotransferase, alkaline phosphatase) and total bilirubin concentration were noted in 8 cats. Four out of 5 of these cats had pathologic evidence of hepatic plasma cell infiltration (either ante or post mortem). Hypercalcemia (total serum calcium) was noted in 2 cats. Hyperglobulinemia was defined as $\geq 5.2 \mathrm{~g} / \mathrm{dL}$ (reference range, 2.6$5.1 \mathrm{~g} / \mathrm{dL}$ ). Hyperglobulinemia was observed at initial presentation in 17 cats (median, $9.1 \mathrm{~g} / \mathrm{dL}$; range, 5.6$16.9 \mathrm{~g} / \mathrm{dL}$ ). One additional cat developed hyperglobulinemia 125 days after excisional biopsy of an intestinal NCEMP. In cats with suspected HVS $(\mathrm{n}=6)$, serum total globulin (mean $\pm \mathrm{SD}, 10.8 \pm 4.5 \mathrm{~g} / \mathrm{dL}$ ) was higher than that in cats without compatible signs (mean $\pm \mathrm{SD}$, $8.5 \pm 2.5 \mathrm{~g} / \mathrm{dL})$, but not significantly $(P=.26)$. SPE was performed in all hyperglobulinemic cats and $100 \%$ had paraproteinemia ( $\mathrm{n}=15$ monoclonal, $\mathrm{n}=3$ biclonal). SPE was performed in 1 normoglobulinemic cat after incomplete excision of multiple skin tumors but it did not identify a paraprotein. In 7 cats, radial gel immunodiffusion identified the serum paraprotein to be immunoglobulin $\mathrm{G}(\mathrm{IgG})$, and in all cats this finding agreed with the immunohistochemistry findings. Urinary immunoglobulin light chains (UILC) were detected in 7 of 16 cats tested by UPE. Five cats in which UILC were detected also had urine protein:creatinine ratio (UPC) determined. Only 2 cats had an abnormally high UPC, but in both cats it was $<5$, implying that there was not marked proteinuria as may occur with concurrent renal amyloidosis.

\section{Correlation of Histopathologic Lesions and Imaging Results}

None of the cats presenting with lameness or pain had radiographic evidence of bony lesions. In total, 13 cats had radiography performed (4 complete and 9 partial skeletal surveys), and an osteolytic lesion was observed in 1 cat. A $z$-test demonstrated that the difference in the proportion of MRD cases with radiographically detectable bone lesions between cats $(8 \%)$ and humans $(80 \%)^{7}$ was significantly different $(P<.001)$.

Twelve cats had abdominal imaging performed (radiography $\mathrm{n}=11$, ultrasound $\mathrm{n}=8)$. Hepatic $(\mathrm{n}=$ $8)$ and splenic $(\mathrm{n}=5)$ abnormalities (typically organomegaly or abnormalities in echotexture) were common. In total, $85 \%$ of those abdominal organs with an imaging abnormality were shown to have plasma cell infiltration within that tissue.

Thirteen cases had thoracic imaging performed (radiography $\mathrm{n}=13$, echocardiography $\mathrm{n}=3$ ). Cardiomegaly was observed in 4 cats and attributed to hypertrophic cardiomyopathy (HCM) in 2 cats and to HCM with pericardial effusion in 1 other cat. Pericardiocentesis yielded a sterile hemorrhagic exudate, with no evidence of plasma cells in this cat. A diffusely increased bronchointerstitial pattern was noted on thoracic radiographs in 2 cats.

\section{Correlation of Histopathologic Lesions and Clinical Results}

In total, $67 \%(\mathrm{n}=16)$ of cats with MRD had pathologically confirmed, marked extramedullary involvement (abdominal organ enlargement or mass identified by palpation or imaging, or presence of a skin mass) at initial presentation. A $z$-test demonstrated that the difference in the proportion of MRD cases with extramedullary involvement at initial presentation between cats $(67 \%)$ and humans $(5 \%)^{11,12}$ was significant $(P$ $<$.001). Plasma cell neoplasia of an abdominal organ, primarily the liver or spleen accounted for $50 \%$ of cats with MRD at initial presentation and increased to $62.5 \%$ at postmortem examination. Cats with abdominal organ 
Table 2. Correlation of lesions with clinical signs, hyperglobulinemia, and multiple organ involvement in cats with MRD. Clinical signs were those of sustained systemic illness such as lethargy, decreased appetite, weight loss, polydipsia etc (as opposed to local signs such as mechanical lameness due to a limb mass) and occurred either at presentation or later in the clinical course of the disease. Serum total globulin concentration and tissue infiltration by plasma cells were also determined at any time point from presentation to time of death.

\begin{tabular}{|c|c|c|c|c|c|c|}
\hline Cases & & $\begin{array}{l}\text { Systemic } \\
\text { Clinical } \\
\text { Signs }\end{array}$ & Hyperglobulinemia & $\begin{array}{l}\text { Abdominal Organ } \\
\text { Involvement }\end{array}$ & $\begin{array}{l}\text { Bone Marrow } \\
\text { Involvement }\end{array}$ & Skin Involvement \\
\hline \multirow{3}{*}{$\begin{array}{l}\text { Bone marrow } \\
\qquad(\mathrm{N}=10)\end{array}$} & $\%+\mathrm{ve}$ & 100 & 90 & 50 & 100 & 0 \\
\hline & $\%-\mathrm{ve}$ & 0 & 0 & 0 & 0 & 100 \\
\hline & $\% \mathrm{NE}$ & 0 & 10 & 50 & 0 & 0 \\
\hline \multirow[t]{3}{*}{$\begin{array}{l}\text { Abdominal organ } \\
\qquad(\mathrm{N}=12)\end{array}$} & $\%+v e$ & 100 & 100 & $\begin{array}{c}75 \% \text { multi-organ, } 25 \% \\
\text { apparently single } \\
\text { organ }\end{array}$ & 42 & 0 \\
\hline & $\%-\mathrm{ve}$ & 0 & 0 & & $\begin{array}{c}42(\mathrm{n}=5 \\
\text { NCEMP })\end{array}$ & 100 \\
\hline & $\% \mathrm{NE}$ & 0 & 0 & 0 & 16 & 0 \\
\hline \multirow[t]{3}{*}{$\begin{array}{c}\text { Skin/subcutis } \\
(\mathrm{N}=7)\end{array}$} & $\%+v e$ & 29 & 29 & 0 & 0 & $\begin{array}{c}57 \% \text { single mass, } 43 \% \\
\text { multiple masses }\end{array}$ \\
\hline & $\%-\mathrm{ve}$ & 71 & 42 & 0 & 0 & 0 \\
\hline & $\% \mathrm{NE}$ & 0 & 29 & 100 & 100 & 0 \\
\hline
\end{tabular}

MRD, myeloma-related disorders; $\%+v e$, percentage of cases positive for tissue infiltration by neoplastic plasma cells (by cytology and/or histopathology); $\%$-ve, percentage of cases negative; $\%$ NE, percentage of cases not examined. It is assumed that all 24 cats underwent a full integumentary examination; NCEMP, noncutaneous extramedullary plasmacytoma (ie, soft tissue plasma cell neoplasia with negative bone marrow involvement was seen in 5 cases).

involvement $(\mathrm{n}=12)$ were systemically ill, hyperglobulinemic, and generally had plasma cell infiltration in multiple rather than single organs (Table 2). In this group, concurrent bone marrow infiltration was seen in half of the cats examined.

In total, 10 cats with MRD had bone marrow plasma cell infiltration. A cytologic diagnosis $(\mathrm{n}=5)$ was made if representative slides contained $>20 \%$ plasma cell infiltration. A histopathologic diagnosis $(\mathrm{n}=5)$ was made if representative bone core sections contained $>20 \%$ plasma cell infiltration and displayed additional features such as plasma cell clustering, morphologic abnormalities, and immunohistochemical demonstration of single immunoglobulin isotype expression. Cats with bone marrow infiltration $(\mathrm{n}=10)$ were systemically ill, and 90\% were hyperglobulinemic (Table 2). Of those cats that underwent additional tissue examinations, all had evidence of plasma cell infiltration in abdominal organs. Cats with bone marrow or abdominal organ plasma cell neoplasia did not have cutaneous masses.

In contrast to cats with abdominal organ or bone marrow infiltration, 5 of 7 cats with skin masses were not systemically ill or hyperglobulinemic. One cat with a single mass and 1 cat with multiple skin masses had evidence of systemic involvement and were hyperglobulinemic (Table 2).

\section{Treatment, Outcome, and Survival}

The median number of days from the first reported onset of clinical signs to pathologic diagnosis was 15 days (range, 2-206 days) in those cats with skin masses and 31 days (range, 5-270 days) in cats with abdominal or bone marrow involvement. A diversity of treatments and small groups negated the value of statistical comparison. Cats with skin masses that were not treated $(\mathrm{n}=1)$ or that were given steroids only $(\mathrm{n}=$ 2) had a median survival of 93 days from diagnosis (range, 69-230 days). By contrast, those that underwent excision of the skin masses $(n=4)$ had more prolonged survival (median survival, 663 days; range, 18880 days).

Cats with abdominal MRD, bone marrow MRD, or both, that were not treated $(\mathrm{n}=5)$ or given steroids only $(\mathrm{n}=3)$, deteriorated rapidly (median survival, 5 days; range, 0-98 days). Three cats received melphalan and prednisolone. Administration of a melphalan $2 \mathrm{mg}$ tablet PO (approximately $8 \mathrm{mg} / \mathrm{m}^{2}$ every 4 days) was associated with episodes of marked neutropenia in 2 cats, leading to early euthanasia in 1 cat. Cats that received lower dosages (approximately $2 \mathrm{mg} / \mathrm{m}^{2}$ every 4 days) did not experience neutropenic episodes or illness. Four cats received combination chemotherapy (combinations of cyclophosphamide, vincristine, prednisolone, chlorambucil, and melphalan). For all chemotherapies (excluding use of steroids alone), 6 of the 7 cats improved clinically and had reduced globulinemia (median reduction in serum globulin concentration $42 \%$; range, $21-57 \%$ ) with normoglobulinemia achieved in 3 cats. Rapid reductions in serum globulin concentration were observed (in 1 cat, serum globulin concentration halved within 14 days after beginning chemotherapy). For all chemotherapies, median survival time was 284 days (range, 9 days to 22 months). The subgroup of responders to chemotherapy (those that improved clinically and had reduced globulinemia) had an in- 
creased median survival of 373 days from diagnosis (range, 8.5-22 months).

Two cats underwent surgery and did not receive steroids or other chemotherapeutics. After splenectomy, 1 cat improved clinically and achieved normoglobulinemia but later was euthanized 565 days after diagnosis, due to general deterioration. The second cat underwent partial intestinal resection and improved clinically, but later developed worsening paraproteinemia and was euthanized at 137 days.

Overall, $25 \%$ of the 24 cats in this series were euthanized or died around the time of presentation without treatment. Six of 7 cats with skin masses were euthanized because of general deterioration or other unspecified reason. One cat was still alive 680 days after incomplete excision of skin masses. Approximately $40 \%$ of those with bone marrow or abdominal organ MRD that began treatment were later euthanized because of general deterioration, often accompanied by increasing serum globulin concentrations. The remainder died or were euthanized because of seizures, severe dyspnea, hindlimb paralysis, terminal renal failure, or unspecified causes.

\section{Association with Other Diseases and Vaccination}

Many cats were examined for the presence of infectious diseases including antibody to FIV $(\mathrm{n}=12$, enzyme-linked immunosorbent assay [ELISA] snaptest) and FeLV antigen $(\mathrm{n}=11$, ELISA snaptest) all with negative results. Antibodies to feline coronavirus were assessed in 9 cats and were either negative or of low titer. The medical history (including complete referral and primary veterinarian records from the owner's acquisition of the cat until MRD diagnosis) was assessed for evidence of historic or concurrent chronic antigenic stimulation in all 24 cats. Severe, recurrent gingivitis and periodontitis were identified in 1 cat and unilateral uveitis of unknown etiology in another (3.5 years before the diagnosis of MRD in this cat). There was no other evidence of chronic antigenic stimulation or inflammation preceding the development of MRD. Patient vaccination history was also examined. Two cats had never been vaccinated, whereas the remainder had been vaccinated against calicivirus, herpes, and panleukopenia viruses with some also vaccinated against FeLV. A diversity of vaccines was used, and there was no evidence of a temporal relationship between the last administration of a vaccine (from days to $>10$ years) and the commencement of clinical signs associated with MRD. No cat had evidence of nonplasma cell tumor development in their past medical history, or after diagnosis of MRD.

\section{Discussion}

The literature on MRD in cats comprises case reports and 3 small case series with $\leq 9$ cats. ${ }^{13,20,22}$ These reports outline a diversity of MRD presentation and neoplastic behavior, with sometimes confusing terminology. Cases have been reported as MM despite pathologic evidence of absence of bone marrow infiltration, instead having plasma cell tumors in other locations (EMP).$^{14}$ Con- versely, a review of the literature identified only a single case of MM with bone marrow infiltration and no other organ involvement evident after full postmortem examination. ${ }^{23}$ Indeed, the combination of medullary and extramedullary neoplasia or extramedullary neoplasia alone is widespread among the reported cases of MRD in cats. In humans, extramedullary involvement can be found in two thirds of patients with MM at autopsy, ${ }^{11}$ but only rarely $(<5 \%)$ is extramedullary involvement the presenting manifestation of an MRD. ${ }^{11,12}$ The hypothesis was that cats with MRD commonly present with extramedullary involvement in comparison to human patients. This was demonstrated in this series, where the difference in the proportion of cases with extramedullary involvement at initial presentation between cats $(67 \%)$ and humans $(5 \%)^{11,12}$ was significantly different $(P<.001)$.

Historically, there has been a lack of recognition of the differences in biologic behavior of these tumors among different species. For example, in both dogs and humans, cutaneous extramedullary plasmacytoma typically is nonsecretory and has a benign course with surgical excision being curative of an MRD. ${ }^{12,24}$ By contrast, in reports of 4 cats with skin masses all were paraproteinemic, suffered rapid tumor development, and were affected by multi-organ plasma cell infiltration. ${ }^{8}, 18,25,26$ Similarly, in this case series, 2 cats with skin masses were paraproteinemic and had clinical or imaging evidence of internal involvement. Overall, hyperglobulinemia was seen less commonly in cats with skin masses in this study. Surgical excision appeared to be associated with prolonged survival (in comparison with those cats with abdominal organ or bone marrow involvement). This finding suggests that in some cats, at least, nonmetastatic or early CEMP was present. CEMP can, with time, develop into MM in a small proportion of human patients. ${ }^{10,12}$ This phenomenon has rarely been reported in the $\operatorname{dog}^{27}$ or the cat. ${ }^{26}$ For the most part, the cats with skin masses in this series were seen in general practice and further investigations often were limited. In particular, demonstration of the presence or absence of plasma cells in other tissues (eg, bone marrow, internal organs) generally was not carried out (Table 2), so a conclusive diagnosis of CEMP could not be made.

Oral, macroscopically nodular, plasma cell lesions were located bilaterally in the fauces of 1 cat with histopathology and immunohistochemistry confirmed multiple organ (abdominal and bone marrow) involvement and monoclonal paraproteinemia. One other cat with an immunohistochemistry-confirmed oral EMP has been reported, although details of other organ involvement or clinical signs were not described. ${ }^{28}$ Lymphocytic-plasmacytic stomatitis, occasionally, can be associated with mild polyclonal gammopathies and reactive plasma cell lesions may be identified by immunohistochemistry. A monoclonal gammopathy has been reported in 1 cat that did not undergo immunohistochemistry or postmortem examination. ${ }^{29}$

NCEMP is extremely rare in both dogs and humans. In these species, NCEMP behavior is unpredictable, with some neoplasms being apparently benign and 
others aggressive in nature. ${ }^{30-33} \mathrm{By}$ comparison, all of the reported NCEMP in cats have been characterized by signs of systemic disease or metastatic behavior. ${ }^{18,3438}$ Typically, the spleen and liver were infiltrated. Other infiltrated locations have included the kidneys, gastrointestinal tract, lymph nodes, stomach, epidura, orbit, and retroperitoneal space ${ }^{18,34-38}$ In this series, NCEMP was documented in 5 cats $(42 \%$ of cats with abdominal organ involvement had no pathologic evidence of bone marrow infiltration, Table 2). Five cats with abdominal organ neoplasia had concurrent bone marrow infiltration, and it could not be determined if these cats had MM (primary bone marrow involvement) with secondary extramedullary metastases or EMP (primary soft tissue involvement) with secondary bone marrow infiltration. Five of 10 cats with bone marrow infiltration did not undergo additional tissue investigations, and it is not known if other internal organs were involved.

Of the 10 cats with bone marrow involvement, all had marked infiltration ranging from $20-80 \%$ of the total number of myeloid and lymphoid cells examined. In human MM patients, there is no minimum level of clonal bone marrow plasma cell infiltration, because 5\% of patients will have $<10 \%$ plasma cells in the marrow. ${ }^{7}$ Plasma cell infiltration is associated with osteoclastic activation, which results in bone lysis in affected humans and dogs. ${ }^{6,7,39} \mathrm{~A}$ recent retrospective study suggested that MRD in cats is commonly associated with osteolytic lesions. ${ }^{22}$ However, these cats were evaluated at a single practice with an orthopedic bias. The true incidence of osteolysis in MRD in cats is not known. Our multicenter series included cats from a wide variety of medical and surgical disciplines in an attempt to avoid bias, although the conclusions that can be drawn are limited by the retrospective nature of the study. Thirteen cats underwent radiography, and only 1 cat had demonstrable osteolysis. This finding was significantly different from the situation observed in human patients with MRD, of whom approximately $80 \%$ have radiographically detectable bone pathology. ${ }^{7}$ Furthermore, an overview of the literature determined that of 42 cats with MRD reported to have had radiography, only $14(33 \%)$ had radiographically detectable bony lesions. ${ }^{16,19,22,40,41}$ This observation does not take into account the possibility that there may have been a historic bias toward reporting cats with MRD and bone lesions, based on diagnostic criteria used for human patients with MM that have been extrapolated to the cat. A $z$-test comparing the literature-estimated prevalence of radiographic bone lesions in cats with MRD versus the prevalence observed in humans with MRD revealed a significant difference (33\% versus $80 \%, P<.001)$. Based on the medical literature and the statistical analysis of this series, we conclude that the study hypothesis (cats with MRD uncommonly present with radiographic bone lesions in comparison with humans with MRD) is likely to be true.

In contrast to cats presented with skin masses, those with abdominal organ or bone marrow involvement or both shared common clinical, clinicopathologic, and pathologic features. These cats tended to be systemically ill, hyperglobulinemic, and frequently had neoplasia of multiple organs (Table 2).

Records were examined for evidence of secondary pathology consistent with the presence of MRD. HVS can occur in MRD. An absolute diagnosis of HVS requires compatible clinical signs, measurement of blood viscosity, and exclusion of other pathology. ${ }^{42}$ Signs compatible with HVS were observed in 6 cats, including central nervous system signs, fundic changes, and hypertrophic cardiomyopathy. All hyperglobulinemic cats were paraproteinemic, and $83 \%$ showed monoclonality. Three cats showed biclonality, a phenomenon that has been reported previously and is likely to be attributable to the development of 2 neoplastic clones or isotype switching of some clones. ${ }^{9,41}$ Defects in hemostatic function typically are attributable to thromobocytopathia due to hyperglobulinemia or thrombocytopenia. ${ }^{6,15}$ In this series, 4 cats presented with clinical signs of bleeding, but tests of hemostatic function were not carried out. Immunodeficiency has been documented in human myeloma patients ${ }^{43}$ and an increased risk of atypical or recurrent infections has been reported in affected humans and cats. ${ }^{23,40}$ No cats with atypical or recurrent infections were identified in this series.

The etiopathogenesis of MRD in cats has not been explored previously. In this series, there was no evidence of FIV, FeLV, or FIP infection in any of the cats tested. In murine models, chronic antigenic stimulation has been demonstrated to drive or enhance the chromosomal abnormalities that are necessary for the neoplastic transformation of plasma cells. ${ }^{44,45}$ Studies in humans have determined that exposure to substances such as petroleum products, increases the likelihood of developing MM. ${ }^{46}$ Epidemiologic studies were not carried out in this retrospective case series, but examination of the complete past medical records for 24 cats did not identify any significant inflammatory or antigenic stimulatory factor. Vaccination in humans has been hypothesized to trigger the development of $\mathrm{MM} \cdot{ }^{47} \mathrm{In}$ this series, there was no evidence that vaccine type or time of administration influenced development of MRD.

The possibility that human patients with $\mathrm{MM}$ are at increased risk of developing other nonplasma cell neoplasms is controversial. ${ }^{11}$ Examination of the past medical history and complete follow-up for all 24 cats identified no other neoplasms.

In conclusion, this multicenter retrospective study details the clinical, clinicopathologic, and imaging features as well as treatment and outcome of 24 cats affected by this rare neoplasm. The hypotheses that cats with MRD at initial presentation (1) commonly display extramedullary involvement and (2) uncommonly have radiographically detectable bone lesions in comparison with human patients with MRD have been demonstrated. MRD in cats has a wide spectrum of dissemination and frequently displays aggressive neoplastic behavior. Additional careful clinical investigations are warranted. Although some skin masses truly may be CEMP and respond favorably to complete excision, there should be an awareness of the potential for underlying multi-organ 
involvement. In the presence of paraproteinemia, bone marrow aspirates and core biopsy samples should be examined. Abdominal organ involvement, especially of the spleen and liver, is common. In this series, there was good correlation between an abdominal organ abnormality seen at imaging (eg, radiography, ultrasonography) and tissue infiltration. Cats with abdominal involvement or bone marrow infiltration commonly displayed systemic signs of illness, but the majority responded well to chemotherapy and the median survival time of responders was 12.3 months.

\section{Footnotes}

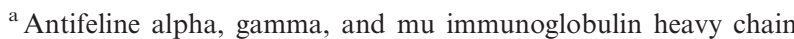
antibodies. Nordic Immunological Laboratories, Tilburg, The Netherlands

${ }^{\mathrm{b}}$ Antihuman lambda and kappa light chain antibodies. DakoCytomation Ltd, Ely, UK

\section{Acknowledgments}

The authors would like to thank the cat owners and our colleagues in first opinion practice, who kindly referred either cases or case material and without whose help these rare diseases could not have been characterized. Many thanks to staff at the following: all $6 \mathrm{UK}$ veterinary schools; the Animal Health Trust; Davies Veterinary Specialists; Wey Referrals; Willows Referrals; North Kent Referrals; North Downs Referrals; Veterinary Referrals Cancer and Critical Care Center; Godiva Referrals; Stone Lion Veterinary Center; Animal Medical Center; Oxford Cat Practice; Nine Lives Referrals; Finn Pathology; Cytopath Ltd; Rest Pathology; IDEXX Laboratories Ltd; TDDS Laboratories; Axiom Laboratories; and Leeds Veterinary Laboratory Ltd. Special thanks to Jodi Miller (Animal Health Trust) and Colin Nixon (Veterinary Pathology Division, University of Glasgow) for assistance with immunohistochemistry and to Dr Fred Heath (Department of Veterinary Medicine, University of Cambridge) for statistical assistance. The financial support of both the University of Cambridge Veterinary School Trust and Pet Plan Charitable Trust is gratefully acknowledged. Finally, many thanks to Hills Pet Nutrition Ltd who kindly sponsored Paul Mellor's clinical scholarship at the University of Cambridge.

\section{References}

1. Carpenter JL, Andrews LK, Holzworth J. Tumours and tumour-like lesions. In: Holzworth J, ed. Diseases of the Cat: Medicine and Surgery. Philadelphia, PA: WB Saunders; 1987:406-596.

2. Engle GC, Brody RS. A retrospective study of 395 feline neoplasms. J Am Anim Hosp Assoc 1969;5:21-31.

3. Priester WA. The occurrence of tumours in domestic animals. NCI monograph 1980;54.
4. Longo DL. Plasma cell disorders. In: Fauci AS, ed. Harrison's Principles of Internal Medicine, 14th ed. New York, NY: McGraw-Hill; 1998:712-718.

5. The UK Myeloma Forum. Guideline diagnosis and management of multiple myeloma. Br J Haematol 2001;115:522-540.

6. Vail DM. Plasma cell neoplasms. In: Withrow SJ, MacEwen EG, eds. Small Animal Clinical Oncology, 3rd ed. Philadelphia, PA: WB Saunders; 2001:626-638.

7. International Myeloma Working Group. Criteria for the classification of monoclonal gammopathies, multiple myeloma and related disorders: A report of the International Myeloma Working Group. Br J Haematol 2003;121:749-757.

8. Carothers MA, Johnson GC, DiBartola SP, et al. Extramedullary plasmacytoma and immunoglobulin-associated amyloidosis in a cat. J Am Vet Med Assoc 1989;195:1593-1597.

9. Larsen AE, Carpenter JL. Hepatic plasmacytoma and biclonal gammopathy in a cat. J Am Vet Med Assoc 1994;205:708-710.

10. Alexiou C, Kau RJ, Dietzfelbinger H, et al. Extramedullary plasmacytoma: Tumor occurrence and therapeutic concepts. Cancer 1999;85:2305-2314.

11. Kapadia SB. Multiple myeloma: A clinicopathologic study of 62 consecutively autopsied cases. Medicine (Baltimore) 1980;59:380-392.

12. Soutar R, Lucraft H, Jackson G, et al. Guidelines on the diagnosis and management of solitary plasmacytoma of bone and solitary extramedullary plasmacytoma. $\mathrm{Br} \mathrm{J}$ Haematol 2004; 124:717-726.

13. MacEwen EG, Hurvitz AI. Diagnosis and management of monoclonal gammopathies. Vet Clin North Am Small Anim Pract 1977;7:119-132.

14. Mills JN, Eger CE, Robinson WF, et al. A case of multiple myeloma in a cat. J Am Anim Hosp Assoc 1982;18:79-82.

15. Forrester SD, Greco DS, Relford RL. Serum hyperviscosity syndrome associated with multiple myeloma in two cats. J Am Vet Med Assoc 1992;200:79-82.

16. Hay LE. Multiple myeloma in a cat. Aust Vet Pract 1978;8:45-48.

17. Jacobs T. Multiple myeloma in a cat with paraparesis. Feline Pract 1994;22:28-32.

18. Harbison ML. Plasma cell myeloma and plasmacytoma. In: Holzworth J, ed. Diseases of the Cat - Medicine and Surgery. Philadelphia, PA: WB Saunders; 1987:442-444.

19. Sheafor SE, Gamblin RM, Couto CG. Hypercalcaemia in two cats with multiple myeloma. J Am Anim Hosp Assoc 1996;32:503-508.

20. Drazner FH. Multiple myeloma in the cat. Comp Cont Educ Pract Vet 1982;4:206-216.

21. King AJ, Davies DR, Irwin PJ. Feline multiple myeloma: Literature review and four case reports. Aust Vet Pract 2002;32:146-151.

22. Hanna F. Multiple myelomas in cats. J Feline Med Surg 2005; 7:275-287.

23. Weber NA, Tebeau CS. An unusual presentation of multiple myeloma in 2 cats. J Am Anim Hosp Assoc 1998;34:477-483.

24. Clark GN, Berg H, Engler SJ, et al. Extramedullary plasmacytomas in dogs: Results of surgical excision in 131 cases. J Am Anim Hosp Assoc 1992;28:105-111.

25. Dust A, Norris AM, Valli VEO. Cutaneous lymphosarcoma with IgG immunoglobulin monoclonal gammopathy, serum hyperviscosity and hypercalcaemia in a cat. Can Vet J 1982;23:235-239.

26. Radhakrishnan A, Risbon RE, Patel RT, et al. Progression of a solitary, malignant cutaneous plasma-cell tumour to multiple myeloma in a cat. Vet Comp Oncol 2004;2:36-42.

27. Lester SJ, Mesfin GM. A solitary plasmacytoma in a dog with progression to a disseminated myeloma. Can Vet $\mathbf{J}$ 1980;21:284-286. 
28. Majzoub M, Breuer W, Platz SJ, et al. Histopathologic and immunophenotypic characterization of extramedullary plasmacytomas in 9 cats. Vet Pathol 2003;40:249-253.

29. Lyon KF. Feline lymphoplasmacytic stomatitis associated with monoclonal gammopathy and Bence-Jones proteinuria. J Vet Dent 1994;11:25-27.

30. Meis JM, Butler JJ, Osborne BM, et al. Solitary plasmacytomas of bone and extramedullary plasmacytomas: A clinicopathologic and immunohistochemical study. Cancer 1987;59: 1475-1485.

31. MacEwen EG, Patnaik AK, Johnson GF, et al. Extramedullary plasmacytoma of the gastrointestinal tract in 2 dogs. J Am Vet Med Assoc 1984;184:1396-1398.

32. Homma K, Ihzumi $\mathrm{T}$, Nemoto $\mathrm{K}$, et al. Primary extramedullary plasmacytoma of the small intestine. Int $\mathrm{J}$ Hematol 1992;56:179-184.

33. Trevor PB, Saunders GK, Waldron DR, et al. Metastatic extramedullary plasmacytoma of the colon and rectum in a dog. J Am Vet Med Assoc 1993;203:406-409.

34. Michau TM, Proulx DR, Rushton SD, et al. Intraocular extramedullary plasmacytoma in a cat. Vet Ophthalmol 2003;6:177-181.

35. Williams DA, Goldschmidt MH. Hyperviscosity syndrome with IgM monoclonal gammopathy and hepatic plasmacytoid lymphosarcoma in a cat. J Small Anim Pract 1982;23:311-323.

36. Ward DA, McEntee MF, Weddle DL. Orbital plasmacytoma in a cat. J Small Anim Pract 1997;38:576-578.

37. Mandel NS, Esplin DG. A retroperitoneal extramedullary plasmacytoma in a cat with monoclonal gammopathy. J Am Anim Hosp Assoc 1994;20:603-608.
38. Zikes CD, Spielman B, Shapiro W, et al. Gastric extramedullary plasmacytoma in a cat. J Vet Intern Med 1998;12:381-383.

39. Terpos E, Politou M, Rahemtulla A. New insights into the pathophysiology and management of bone disease in multiple myeloma. Br J Haematol 2003;123:758-769.

40. Hawkins EC, Feldman BF, Blanchard PC. Immunoglobulin A myeloma in a cat with pleural effusion and serum hyperviscosity. J Am Vet Med Assoc 1986;188:876-878.

41. Bienzle D, Silverstein DC, Chaffin K. Multiple myeloma in cats: Variable presentation with different immunoglobulin isotypes in 2 cats. Vet Pathol 2000;37:364-369.

42. Mehta J, Singhal S. Hyperviscosity syndrome in plasma cell dyscrasias. Semin Thromb Hemost 2003;29:467-471.

43. Sirohi B, Powles R. Multiple myeloma. Lancet 2004;363:875-887.

44. Janz S, Muller J, Shaughnessy J, et al. Detection of recombinations between c-myc and immunoglobulin switch alpha in murine plasma cell tumors and preneoplastic lesions by polymerase chain reaction. Proc Natl Acad Sci U S A 1993;90:7361-7365.

45. Potter M, Mushinski EB, Wax JS, et al. Identification of 2 genes on chromosome 4 that determine resistance to plasmacytoma induction in mice. Cancer Res 1994;54:969-975.

46. Speer SA, Semenza JC, Kurosaki T, et al. Risk factors for acute myeloid leukemia and multiple myeloma: A combination of GIS and case-control studies. J Environ Health 2002;64:9-16; quiz $35-16$.

47. Schattner A, Berrebi A. Several possible causes for multiple myeloma including a vaccination in a single case study. Vaccine 2004;22:2509-2510. 